\title{
DRUGPATH: A new database for mapping polypharmacology
}

Rajeev Jaundoo and Travis J.A. Craddock

\begin{abstract}
While there are existing databases that curate only drug, target, or pathway data for instance, none of these alone are exhaustive. The Drug Gene Pathway (DRUGPATH) meta database was created as a response to the complex treatment required for various diseases including Gulf War IIIness (GWI) and post-traumatic stress disorder (PTSD), where therapy involves using multiple drugs in combination. Here, drug-drug interactions can occur due to the promiscuous nature of pharmaceuticals, which can then lead to various side effects or can alternatively be utilized towards drug repurposing. The objective was to develop a database that maps the interactions between drugs, genes, pathways, and targets for use in the treatment of complex diseases, including the prediction of off-target interactions, otherwise known as side effects. Using MATLAB and Python scripts, interactions between known drugs, genes, targets, and pathways amalgamated from numerous expert-curated sources such as PharmGKB, DrugBank, DGIdb, ConsesusPathDB, Guide to PHARMACOLOGY, HUGO Gene Nomenclature Committee, Toxin and Toxin-Target Database, repoDB, the FDA's National Drug Code database, etc. were mapped together. The raw data was first downloaded from its source and subsequently cleaned, where extraneous information such as data from non-humans, internal identifiers, timestamps, etc. were removed. The remaining information was then integrated into an SQLite database. DRUGPATH currently contains a total of 2,632,516 unique entries, and of these, there are 54,757 unique genes, 2,632,242 unique pathways, and 31,042 unique drugs. DRUGPATH allows researchers and clinicians to discern which pathways are affected by each drug, reducing the likelihood of an adverse drug reaction occurring. The incorporation of drug, gene, target, and pathway information makes DRUGPATH a powerful resource for predicting potential side effects when designing or refining a given drug combination therapy. Not only that, but we have additionally added the FDA status, half-life, and indication for each drug whenever possible for clinical applications of this database.
\end{abstract}

Cite as: Jaundoo R. and Craddock TJA. 2019. DRUGPATH: A new database for mapping polypharmacology. Alberta Academic Review, Vol 2 (3) 4, CASCH Special Issue (not peerreviewed), DOI: 10.29173/aar92. 